\title{
Medium and Long Chain Fat Absorption in Patients with Cirrhosis*
}

\author{
Willem G. Linscheer, † James F. Patterson, Edward W. Moore, Richard J. \\ Clermont, Sander J. Robins, and Thomas C. Chalmers \\ (From the Medical Services of the Lemuel Shattuck Hospital, the Massachusetts Department \\ of Public Health, the Gastroenterology Service of the New England Medical Center \\ Hospitals, and the Department of Medicine, Tufts University School of \\ Medicine, Boston, Mass.)
}

Medium chain fatty acids (MCFA) are known to differ from long chain fatty acids (LCFA) in their absorption, transportation, and metabolism. In malabsorption states, medium chain triglycerides (MCT) are better absorbed than long chain triglycerides (LCT) (1-3) and have been used in the treatment of such conditions. MCFA are transported from the gut mainly by the portal vein as fatty acids, whereas LCFA are transported primarily by the lymphatics in chylomicrons (4-6). Absorbed MCFA are immediately oxidized in the liver, as demonstrated by ${ }^{14} \mathrm{CO}_{2}$ recovery in the expired air of normal volunteers who have received orally administered ${ }^{14} \mathrm{C}$-labeled MCFA (7-9).

Intestinal absorption, transport, and hepatic clearance of MCFA in patients with cirrhosis are of interest for two reasons: 1) Fat malabsorption sufficient to contribute to malnutrition is common in cirrhosis $(10,11)$, and information about the absorption of medium chain fats is lacking. 2) Portacaval shunting of blood and a variable diminution in the capacity of the cirrhotic liver to metabolize MCFA emphasized the need for study of peripheral blood levels after their administration.

\footnotetext{
* Submitted for publication November 10, 1964; accepted May 12, 1966.

A part of this work appeared in abstract form in J. clin. Invest. 1964, 43, 1280.

Investigation was supported by contract DA-49-193MD-2248 and contract DA-49-193-MD-2707 between Tufts University and the Office of the Surgeon General, Department of the Army, Washington, D. C.; by research grants AM-2547 and AM-07417 and training grant AM5424, U. S. Public Health Service; and by appropriation of the Commonwealth of Massachusetts, item 2026-50 of the 1954 General Appropriation Act.

$\dagger$ Address requests for reprints to Dr. Willem G. Linscheer, Lemuel Shattuck Hospital, 170 Morton Street, Boston, Mass. 02130.
}

Balance studies were performed to evaluate the incidence of fat malabsorption in patients with alcoholic cirrhosis admitted to the Lemuel Shattuck Hospital and to select patients with malabsorption for comparative study of the absorption of MCT and LCT. In addition, the absorption rate of a single MCFA (octanoic acid) from a segment of upper small bowel was studied in groups of control and cirrhotic patients. During these studies, serum concentrations of octanoic acid were compared in normals and cirrhotics with and without surgically constructed portacaval shunts.

Absorption of medium chain fats was found to be appreciably better than long chain fats in the patients with cirrhosis, and absorption rate of octanoic acid was equal in controls and cirrhotic patients. Peripheral blood levels of octanoic acid were higher in the cirrhotic patients and related to the amount of blood shunted around the liver.

\section{Methods}

Clinical material consisted of 56 patients. $A$ ) Fat balance studies were performed on: 1) Control group. Six patients who were without clinical or chemical evidence of liver disease, malabsorption, or diabetes composed this group. They were approaching completion of hospitalization for emphysema (2), rheumatoid arthritis, asthmatic bronchitis (3), and ulcerative colitis in remission. 2) Cirrhotic group. This group consisted of 35 patients, 16 of whom had ascites. None of these patients were receiving antibiotic therapy or had previously had a surgical portal-systemic shunt. From this group, ten patients with fecal fats over the normal upper limit of $5 \mathrm{~g}$ per 24 hours were selected for balance studies on LCT- and MCT-containing diets. 3) Shunted group. Nine patients with cirrhosis who had undergone portacaval shunt surgery 1 to 4 years previously composed this group. They had moderate evidence of hepatic decompensation and required continuing hospitalization.

$B$ ) Octanoic acid absorption studies in the upper small bowel were performed on: 1) the previous control group; 


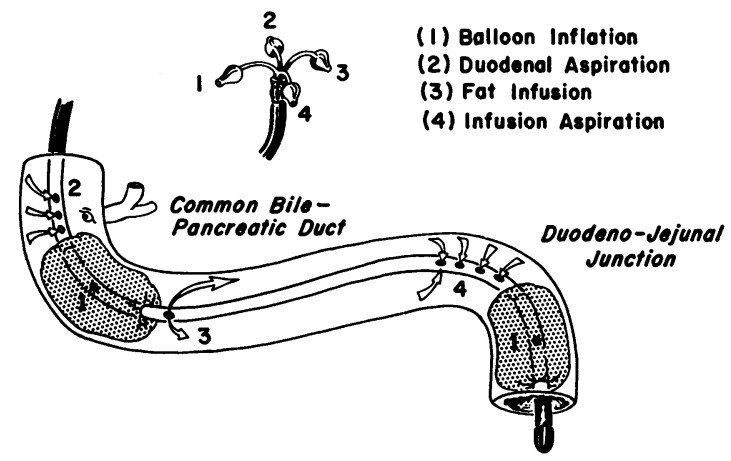

Fig. 1. The FOUR-LUMEN TWO-BALloon tUbE.

2) six patients from the previous cirrhotic group, two of whom had ascites and esophageal varices; 3 ) the previous shunted group, and 4) an additional cirrhotic group that consisted of six patients with variable degrees of decompensation who were chosen merely to test the influence of balloon inflation on absorption as measured by the tube technique.

Dietary fat absorption. In order to measure the absorption of LCT the patients of groups A were placed on a standard hospital diet containing $100 \mathrm{~g}$ fat or on a liquid formula diet containing 90 to $115 \mathrm{~g}$ fat for 6 days on a metabolic ward. The fats of the liquid diet were mainly corn oil, with approximately $15 \%$ in the form of milk fat. The liquid diets were homogenized with a Waring blendor shortly before administration. No difference was observed in fat absorption in six cirrhotic patients during alternating diet periods of 6 days with a regular 100 -g fat diet or the liquid LCT diet [mean 24hour stool fat on the regular diet was $4.5 \pm 1.6$ (SE) $\mathrm{g}$ and on the liquid formula diet, $5.7 \pm 1.6 \mathrm{~g}$ ].

Ten of the patients in the fat balance groups with steatorrhea were given LCT or MCT-liquid diets in alternating periods of 6 days in random order on a metabolic ward. The LCT in eight of the patients consisted of a mixture of oleo oil and vegetable oil ${ }^{1}$ (composition of fatty acids by chain length: $12: 0=1.0 \%, 14: 0=$ $2.2 \%, 16: 0=16.8 \%, 16.1=1.6 \%, 18: 0=9.9 \%, 18.1=$ $53.8 \%, 18: 2=14.3 \%$, and $18: 3=0.6 \%$; total fat $=107 \mathrm{~g}$ per 24 hours). Two patients on a salt-restricted diet received a liquid diet containing corn oil ( 75 to $88 \mathrm{~g}$ ) and milk fat $(15 \mathrm{~g})$. The MCT liquid diets ${ }^{1}$ contained equal caloric amounts of fat and consisted of medium chain triglycerides ( 83 to $86 \%$ ) and long chain triglycerides (14 to $17 \%$ ). Octanoic acid constituted $76 \%$ of the total medium chain fatty acids. The two patients on salt restriction received a MCT liquid diet of comparable composition. The protein and carbohydrates were kept constant during the two balance studies.

Total fat was determined in 72-hour stool collections (fourth, fifth, and sixth days) by the Van de Kamer method (12).

\footnotetext{
${ }^{1}$ Composition and diets supplied by Mea:d Johnson Laboratories, Evansville, Ind.
}

Determination of medium chain fatty acid absorption in the upper small bozvel technique. A four-lumen two-balloon tube (Figure 1) described previously (13) was employed to study absorption rates of octanoic acid in 27.5 $\mathrm{cm}$ of distal duodenum and proximal jejunum without interference from pancreatic juice or the fatty acids of bile. The first lumen of the tube was connected to both balloons. The second lumen was connected to openings just above the proximal balloon so that intestinal contents could be constantly aspirated to prevent intestinal obstruction and contamination of the isolated segment. The third lumen, opening into the upper portion of the segment under study just distal to the proximal balloon, was used for infusion of the test solution, and the infusate was continuously aspirated from the distal end of the loop $27.5 \mathrm{~cm}$ below the infusion point through the fourth lumen. The tube was passed into the small intestine and positioned under fluoroscopic control so that the proximal balloon was just distal to the ampulla of Vater. This was confirmed by constant aspiration of bile through the opening above the proximal balloon.

After the tube was properly positioned, the balloons were inflated to a pressure of 35 to $40 \mathrm{~cm} \mathrm{H}_{2} \mathrm{O}(50 \mathrm{ml}$ of air each), and the segment of intestine was washed with isotonic saline until the washings, initially bile-stained, became colorless. After this, the test solution was infused into the upper end of the loop for 60 minutes by lumen 3 at a constant rate of $6 \mathrm{ml}$ per minute by a Bowman infusion pump while the infusate was continuously aspirated from the lower end of the loop by lumen 4 . After the 60-minute infusion period, the bowel segment was washed with $200 \mathrm{ml}$ isotonic saline infused over a 5-minute period with continuous aspiration.

The four-lumen two-balloon tube has three major advantages: 1) Continuous aspiration above the proximal balloon is effective in preventing contamination of the segment under study with bile and pancreatic or gastric juice, or with octanoic acid, which may be excreted with bile (14) and disturb measurement of true absorption.

2) The second balloon prevents escape of large quantities of test solutions into the distal bowel, thus limiting the absorbing area to proximal small bowel. Serum concentrations of octanoic acid are thus related to absorption from a relatively constant area of small bowel, and absorption can be discontinued by a washing procedure.

3) The relatively low pressure of the balloons does not cause hyperperistalsis and bowel spasm in most patients, but leakage around the balloons can occur. Continuous aspiration of bowel juices above both balloons prevents leakage and also accumulation of stagnating fluids, which might cause discomfort and interfere with absorption. Results in four of 25 patients had to be discarded because of bowel spasm or leakage of bile.

In order to study possible effects of the balloons on absorption, the rate of octanoic acid absorption was measured in six patients (B, additional cirrhotic group) with the balloons collapsed and compared with the rate measured with balloons inflated. During the latter period, as- 
TABLE I

Titration of polyethylene glycol (PEG) and octanoic acid in the duodenal aspirate immediately after mixing and after 3 hours standing without further mixing

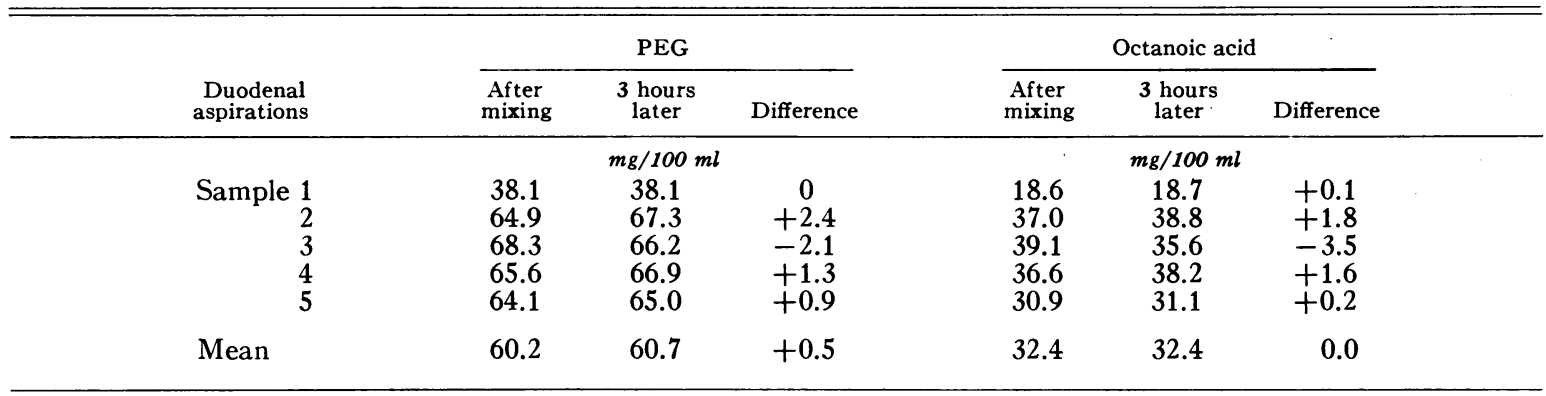

pirated bile and pancreatic juice were reinfused with the test solution.

The infusate, with $\mathrm{pH} 4.72$, contained a micellar solution of octanoic acid $(10 \mathrm{mg}$ per $\mathrm{ml})$, sodium taurocholate ( $30 \mathrm{mg}$ per $\mathrm{ml}$ ), and a nonabsorbable marker, polyethylene glycol (PEG), mol wt 4,000 (10 $\mathrm{mg}$ per $\mathrm{ml}$ ) in $0.85 \% \mathrm{NaCl}$. PEG served as a measure of the net effects of dilution, absorption, and secretion of water and the amount of leakage of the test solution. Commercial octanoic acid ${ }^{2}$ contained only traces of decanoic acid as tested by gas-liquid chromatography and no other fatty acids. Sodium taurocholate ${ }^{3}$ was purified by repeated extraction with ether-heptane-ethanol-water (1:1:1:1 by volume) (15).

A clear micellar solution of octanoic acid was formed due to its solubility in water in the presence of sodium taurocholate above a critical micellar concentration (16). A tenfold dilution with isotonic saline also resulted in a clear solution, as demonstrated turbidimetrically.

Venous blood samples for the determination of octanoic acid by gas-liquid chromatography $(17,18)$ by the Dole method (19) were drawn before infusion of the test solution, at the end of the 60 -minute infusion period, and again at 120 and 180 minutes.

PEG was determined by the method of Hyden (20). Although there is some controversy as to the applicability of PEG in long chain fat absorption studies (21), the stability and water solubility of octanoic acid in the test solutions allowed the use of PEG as a nonabsorbable marker in these studies. Since there was no separation of nonstirred bowel aspirations over a 3-hour period ( $\mathrm{Ta}$ ble I), we concluded that PEG was a reliable marker for absorption studies of octanoic acid in a micellar test solution.

Analysis of octanoic acid. Analysis of octanoic acid in both test solutions and bowel aspirations was performed by the Dole method (19); however, since bile acids are also titrated by this technique, it was necessary to analyze for the infused sodium taurocholate specifically in all bowel aspirations. This was accomplished by the Kier method (22). Sodium taurocholate was expressed in milligrams per milliliter. A straight line correlation was

2 Eastman Organic Chemicals, Rochester, N. Y.

3 Nutritional Biochemicals Corp., Cleveland, Ohio. found between milliequivalents of sodium taurocholate added to bowel juice and milliequivalents $\mathrm{NaOH}$ used for the titration of taurocholic acid extracted by the Dole method $(r=0.9976)$. Octanoic acid concentration in bowel aspiration was thus calculated as the difference between the total acid concentration (octanoic acid + taurocholic acid) as determined by the Dole method and the taurocholic acid concentration.

Figure 2 represents the correlation between titration equivalents of alkali used for titration corrected for varying concentrations of taurocholic acid (determined by the Kier method) and octanoic acid (milligrams per milliliter) added to bowel juice obtained during an infusion with isotonic saline.

Due to its water solubility, octanoic acid was not quantitatively recovered by a single Dole extraction procedure. Recovery of ${ }^{14} \mathrm{C}$-labeled octanoic acid added to small bowel aspirations utilizing a single Dole extraction procedure was $58.0 \pm 0.8 \%$ ( $1 \mathrm{SE}$ ) (Table II). In order to determine the reliability of the titration of fatty acids by Dole's method with $\mathrm{NaOH}$, we labeled a $1 \%$ octanoic acid test solution with a tracer amount of octanoic acid- ${ }^{14} \mathrm{C}$. The test solution and its serial dilutions, ranging from 0.1 to $0.9 \%$, we extracted and titrated by the Dole method. Radioactivity (in the upper layer) was determined in a Packard Tri-Carb scintillation counter using Bray's liquid scintillator (23). Table III shows the close correlation between the two methods.

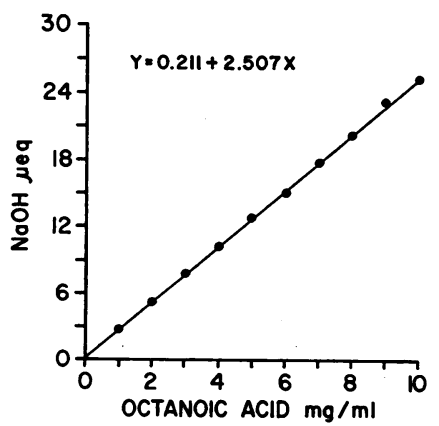

Fig. 2. Titration of AdDed amounts of, octanoic ACID TO BOWEL JUICE BY THE DOLE METHOD, CORRECTED FOR SODIUM TAUROCHOLATE. 
TABLE II

Recovery of ${ }^{14} C$-labeled octanoic acid from a standard heptane solution and duodenal aspirations in a single Dole extraction

\begin{tabular}{rl}
\hline \hline Sample & $\begin{array}{c}\text { Per cent } \\
\text { recovery }\end{array}$ \\
\hline 1 & 57.3 \\
2 & 56.9 \\
3 & 59.5 \\
4 & 56.3 \\
5 & 56.6 \\
6 & 61.5 \\
Mean \pm 1 SE & $58.0 \pm 0.85$ \\
Standard & 58.2 \\
\hline
\end{tabular}

Since the per cent extraction of octanoic acid by Dole's method was constant in test solutions and in bowel juice (Table II), absorption in per cent was calculated by the formula: per cent absorption $=[1-\mathrm{OcAa} \times \mathrm{PEGi} /$ OcAi $\times$ PEGa) $] \times 100$, where OcAa equals the concentration of octanoic acid in the aspirate, PEGi equals the concentration of PEG in the infusate, OcAi equals the concentration of octanoic acid in the infusate, and $\mathrm{PEGa}$ equals the concentration of PEG in the aspirate.

Determination of serum unesterified octanoic acid. A Research Specialties chromatograph with a $\frac{1}{8}$-inch 6-foot U-shaped stainless steel column connected to a hydrogen flame detector was used. The stationary phase was $10 \%$ diethylene glycol succinate on Anakrom ABS, 4 to which had been added $1 \%$ by weight of $85 \%$ orthophosphoric acid $(17,18)$. Standard conditions were: vaporizer temperature $260^{\circ}$, column temperature $180^{\circ}$, nitrogen pressure 20 pounds per inch ${ }^{2}$.

Serum octanoic acid was extracted by the method of Dole. The 3-ml upper layer extract, usually used for

TABLE III

Octanoic acid recovery from standard solution with one Dole extraction*

\begin{tabular}{|c|c|c|c|}
\hline \multirow{2}{*}{$\begin{array}{l}\text { Concen- } \\
\text { trations }\end{array}$} & \multicolumn{2}{|c|}{ Method } & \multirow[b]{2}{*}{ Difference } \\
\hline & $\mathbf{A}$ & B & \\
\hline \multicolumn{4}{|l|}{$\%$} \\
\hline 0.9 & 0.908 & 0.892 & -0.106 \\
\hline 0.8 & 0.801 & 0.792 & -0.009 \\
\hline 0.7 & 0.701 & 0.685 & -0.016 \\
\hline 0.6 & 0.584 & 0.577 & -0.007 \\
\hline 0.5 & 0.486 & 0.497 & +0.011 \\
\hline 0.4 & 0.390 & 0.395 & +0.005 \\
\hline 0.3 & 0.293 & 0.296 & +0.003 \\
\hline 0.2 & 0.193 & 0.191 & -0.002 \\
\hline \multirow[t]{3}{*}{0.1} & 0.100 & 0.114 & +0.104 \\
\hline & \multirow{2}{*}{\multicolumn{2}{|c|}{$\begin{array}{l}\text { Mean } \\
\text { SE }\end{array}$}} & -0.002 \\
\hline & & & 0.018 \\
\hline
\end{tabular}

${ }^{*} \mathrm{~A}=$ recovery of octanoic acid- ${ }^{14} \mathrm{C} ; \mathrm{B}=$ recovery by titration with $\mathrm{NaOH}$. Standard $=1.0 \%$ octanoic acid.

4 Analytic Engineering Laboratories, Hamden, Conn. titration of the free fatty acids, was taken just to dryness in a centrifuge tube under a constant stream of nitrogen at room temperature.

Quantitative determinations were performed by dissolving the dried upper phase from the Dole extraction in $10 \mu \mathrm{l}$ of heptane and applying $5 \mu \mathrm{l}$ of this solution to the column with a Hamilton syringe. Standard pure acid gave a peak with a 3-minute retention time. Column response was standardized during each day's run by multiple applications of a standard solution of octanoic acid $(0.5 \mu \mathrm{g}$ per $\mu \mathrm{l})$ in heptane. Since the same compound was used throughout and the retention time remained constant, peak height rather than peak area was used for quantitation.

It is recognized that there are several sources of loss of octanoic acid in this method. The single Dole extraction does not totally extract octanoic acid from plasma. However, in 100 single Dole extractions of aqueous sodium octanoate solutions at concentrations of 20 and 40 $\mathrm{mEq}$ per $\mathrm{L}$ the percentage recovery, measured by titration of the fatty acid as compared to palmitic acid (which is the standard used in the Dole method), was quite constant $(59.6 \pm 0.07)$. Since octanoic acid is volatile, some loss undoubtedly occurred during the process of evaporating the Dole extract to dryness. Only $5 \mu 1$ of the 10 $\mu 1$. of heptane in which the octanoic acid was redissolved was injected into the gas chromatograph. By keeping the tube in ice to reduce evaporation the injected proportion was kept fairly constant.

In order to assess and correct for the above factors, which would prevent direct quantitative estimation of octanoic acid and might introduce variability in its determination, a set of standard sera was used. An aqueous solution of sodium octanoate $(\mathrm{pH} 7.5)$ was added to normal human fasting serum, and serial dilutions with normal serum were made so that ten serum specimens containing each of the following concentrations of added sodium octanoate were obtained: $0,1,5,10,20,40$, and

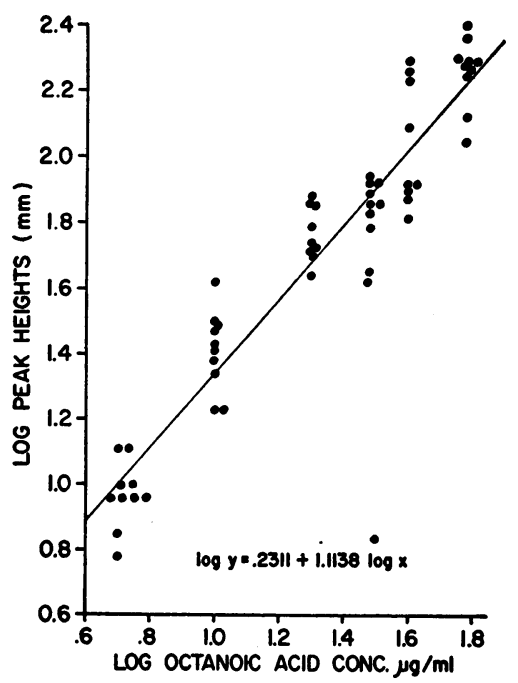

Fig. 3. The determination of octanoic aCid added to NORMAL SERUM BY GAS-LIQUID CHROMATOGRAPHY. 


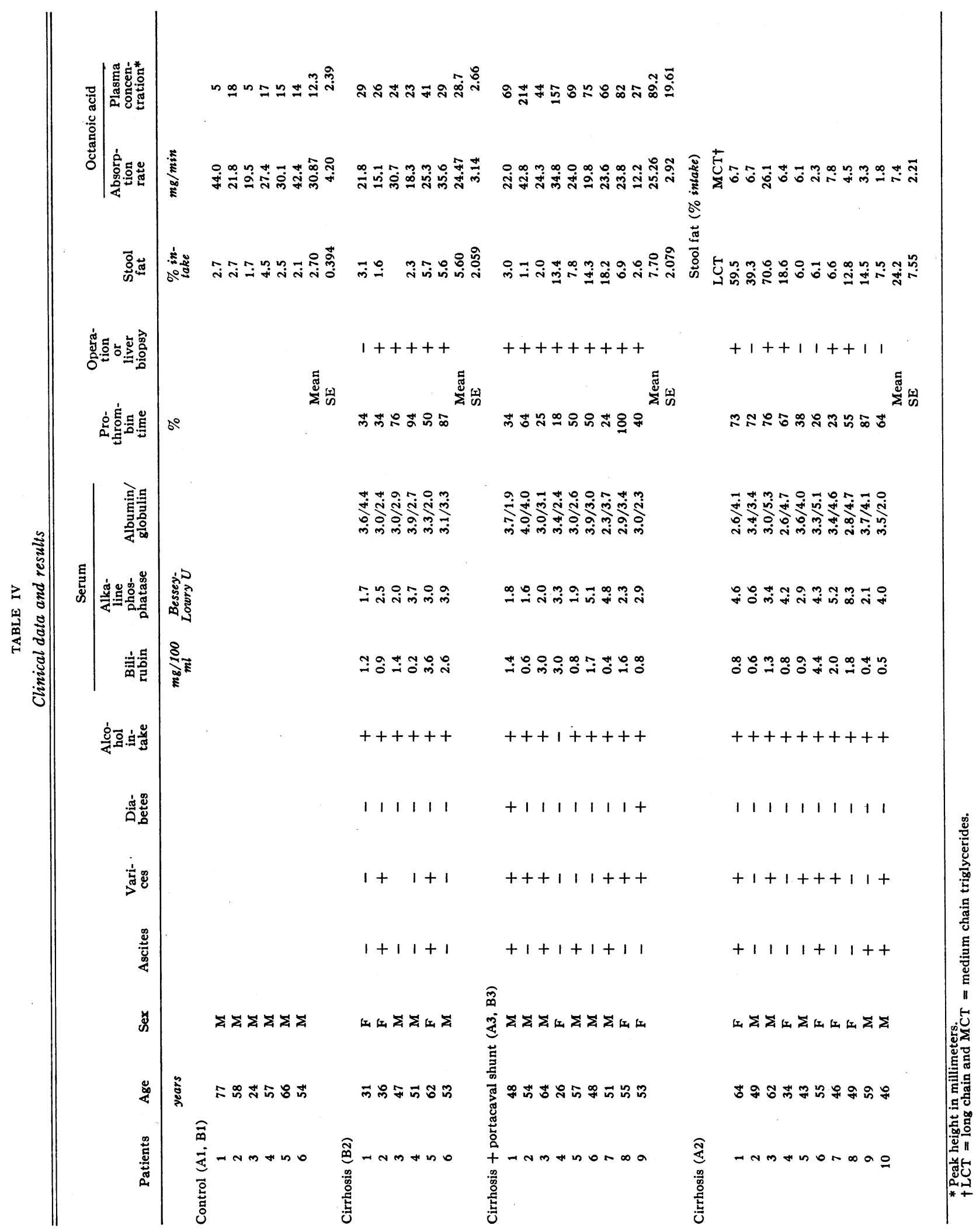


$60 \mu \mathrm{g}$ per $\mathrm{ml}$. The 80 specimens were placed in separate tubes and numbered randomly so that the technician performing the determination was unaware of their content. These samples were interspersed with the duplicate sera. Results, expressed as peak height, are presented in Figure 3. Peak height was linearly related to sodium octanoate concentration on a log-log plot. On this basis, it was felt that the various possible sources of error in the method (extraction ratio, evaporation loss, injected proportion on chromatograph) were constant enough to make the determination useful in the present experiments.

\section{Results}

\section{Fat balance studies}

LCT absorption in noncirrhotic and cirrhotic patients. The absorption of LCT during fat balance studies on a metabolic ward was measured in noncirrhotic patients (group A1) and in cirrhotic patients with and without portacaval shunts (groups A3 and A2). The amount of fat in 72hour stool collections was expressed in Table IV as the average excretion per 24 hours. All six patients of the noncirrhotic group had normal fat excretion. Nineteen $(54 \%)$ of 35 patients with alcoholic cirrhosis of the liver (group A2) excreted more than $5 \%$ of dietary fat per day (mean $=16.7 \pm 4.3 \% \mathrm{SE})$; seven patients $(20 \%)$ had more than $10 \%$ of dietary fat in the feces (mean $=33.2 \pm 9.0 \%$ ). None of these patients received antibiotic treatment. The presence of ascites in 16 patients did not affect fat absorption, and no correlation was shown between liver function and the clinical condition of these patients. The mean fat excretion in all 35 patients was $10.5 \pm 2.6 \%$. Mean fat excretion in nine patients with a portacaval shunt, of whom seven were treated with $\mathrm{Neo}-$

TABLE V

The effect of inflated balloons on the absorption rate of octanoic acid in the upper small bowel*

\begin{tabular}{|c|c|c|c|}
\hline \multirow[b]{2}{*}{ Patient } & \multicolumn{2}{|c|}{ Absorption rate } & \multirow[b]{2}{*}{ Difference } \\
\hline & $\begin{array}{l}\text { Balloons } \\
\text { inflated }\end{array}$ & $\begin{array}{l}\text { Balloons } \\
\text { deflated }\end{array}$ & \\
\hline \multicolumn{4}{|c|}{$m g / \min$} \\
\hline 1 & $17.5 \pm 2.9(\mathrm{SE})$ & $18.0 \pm 2.3(\mathrm{SE})$ & +0.5 \\
\hline 2 & $15.7 \pm 1.6$ & $17.5 \pm 0.7$ & +1.8 \\
\hline 3 & $23.1 \pm 1.7$ & $21.9 \dagger$ & -1.2 \\
\hline 4 & $17.6 \pm 0.2$ & $18.9 \pm 0.8$ & +1.3 \\
\hline 5 & $25.8 \pm 2.3$ & $30.6 \pm 1.8$ & +4.8 \\
\hline 6 & $29.2 \pm 5.3$ & $25.4 \pm 4.4$ & -3.8 \\
\hline Mean & 21.5 & 22.1 & $+0.6 \pm 1.2(\mathrm{SE})$ \\
\hline
\end{tabular}

* When the balloons were inflated, the duodenal aspirate collected during the previous 10 -minute period was added to the infusate.
+ Only one, instead of three, 10 -minute collection periods mycin, was $7.7 \pm 2.1 \%$. The difference between controls and both cirrhotic groups was significant ( $\mathrm{p}<0.01 \mathrm{~A} 1-\mathrm{A} 2 ; \mathrm{p}<0.05 \mathrm{~A} 1-\mathrm{A} 3)$.

Comparison of the absorption of LCT and MCT in patients with cirrhosis and steatorrhea (Table $I V)$. Ten patients (group A2) with cirrhosis of the liver and steatorrhea who were not receiving antibiotic treatment were studied on alternating liquid formula diets containing LCT and MCT for 6 days each. Clinical data and stool fats are expressed in Table IV. Fat absorption improved in eight of the ten patients and did not change in two patients $(\mathrm{p}<0.05)$. (Mean fat excretion: LCT diet $24.2 \%$, MCT diet $7.4 \%$.) Striking results were obtained in patients with severe steatorrhea. Two patients not included in this Table, however, did not tolerate the MCT diet and developed profuse diarrhea with increased stool fat excretion. (Mean fat excretion: LCT diet $12.4 \%$, MCT diet $32.0 \%$.)

\section{Absorption studies in the upper small bowel}

Precision of the four-lumen two-balloon tube technique. The absorption studies were carried out in a "clean" segment of upper small bowel because LCFA and bile acids present in bile and also pancreatic juice may affect absorption of MCFA. Standardization of bile flow rate and pancreatic excretion was impossible, and a segment of small bowel was therefore isolated by means of inflatable balloons. This technique also made it possible to correlate the absorption of octanoic acid in a certain segment of small bowel with octanoic acid levels in the systemic circulation and to study the disappearance from plasma after a rather abrupt discontinuation of the absorptive process by washing with isotonic saline.

To study a possible effect of the balloons on absorption, we measured the rate of octanoic acid absorption in six cirrhotic patients with the balloons collapsed and compared it with the rate measured with balloons inflated. During the latter period, the aspirated bile and pancreatic juice were reinfused with the test solution. Table $\mathrm{V}$ indicates that no consistent error was introduced by inflation of the balloons.

The usefulness of the balloons for recovery of the infusate was shown by the recovery of the total amount of infused PEG $(93.6 \% \pm 5.8 \mathrm{SD})$ in 77 infusion studies. 
The concentration of PEG in 86 bowel aspirations was $67.57 \pm 8.62$ (SD) \% of the concentration of the infusate, and the concentration of sodium taurocholate was $67.24 \pm 8.4 \%$ of the infusate, indicating influx of water into the bowel segment. The concentrations of PEG and sodium taurocholate in the aspirations were closely related $(r=0.8657)$. These findings suggest good mixing of infusate and bowel juice. Furthermore, sodium taurocholate was apparently not absorbed in the upper small bowel since the ratios of the concentrations of PEG/taurocholate were equal in the infusate $(0.333)$ and in the bowel aspirations $(0.335 \pm 0.024 \mathrm{SD})$. Deflation of the balloons decreased the PEG/taurocholate ratio (0.294 \pm 0.031 ) in 39 aspirations because of acids present in bile.

The $\mathrm{pH}$ of the infusate was 4.72 and the average $\mathrm{pH}$ of 100 bowel aspirations was $5.68 \pm 0.69$ (SD).

Rate of absorption of octanoic acid in noncirrhotic and cirrhotic patients (Table $I V$ ). The rate of absorption of a micellar solution of octanoic acid was equal in the control and cirrhotic patients (controls 30.87, cirrhotics 24.86, mg per minute). No difference of absorption was observed between the cirrhotic patients with or without a portacaval shunt.

Plasma octanoic acid levels in relation to small bowel infusion with octanoic acid (Table IV)

No octanoic acid could be detected by gas chromatography in the fasting blood samples in either control or cirrhotic patients (groups B1-3). At the end of the 60-minute infusion of the upper small bowel, small amounts of octanoic acid could be demonstrated in the noncirrhotic group. Mean peak height in the control patients (B1) was $12.3 \pm 2.4$ (1 SE) $\mathrm{mm}$. Significantly higher levels were observed in the group with cirrhosis (B2), $28.7 \pm$ $2.7 \mathrm{~mm}(\mathrm{p}<0.01) .^{5}$ In the shunted patients (group B3) a mean of $89.2 \pm 19.6 \mathrm{~mm}$ was significantly greater than in either group $B 1(p<$ $0.01)$ or group B2 $(p<0.01) .{ }^{5}$ The effect of surgical shunts on plasma octanoic acid levels was clearly demonstrated in group B3. Figure 4 shows the plasma concentration of a 3-hour period. In groups B1 and B2 plasma levels returned to negligible fasting levels within 60 minutes after

\footnotetext{
5 Wilcoxon rank sum test.
}

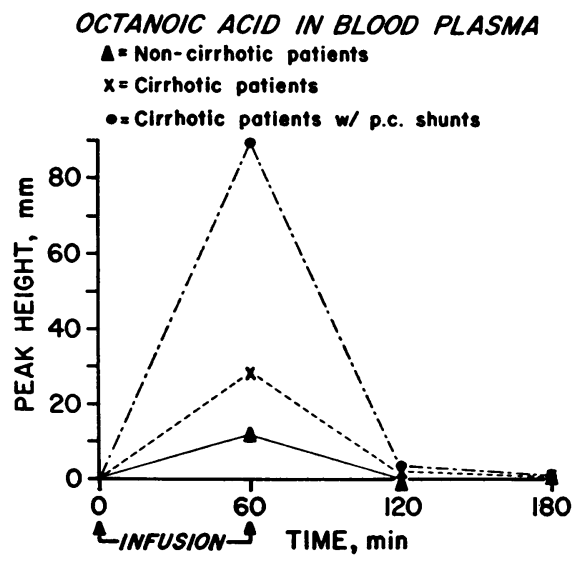

Fig. 4. Plasma levels of octanoic acid before, DURING, AND AFTER INFUSION OF OCTANOIC ACID IN THE UPPER SMALL BOWEL. PC $=$ portacaval.

the infusion period, whereas in the shunted patients (B3) the mean peak heights at this time were $2.6 \pm 0.9 \mathrm{~mm}$.

\section{Discussion}

With the development of more effective therapies for hepatic encephalopathy and gastrointestinal hemorrhage, malnutrition has become one of the major problems in the management of patients with advanced cirrhosis. About $20 \%$ of such patients have considerable steatorrhea. The etiology of this malabsorption is not clear, and no correlation between pancreatic insufficiency and fat malabsorption has been shown $(10,11)$.

The present studies indicate that the administration of medium chain fats may provide a significant source of calories in such patients. Stool fat content was substantially reduced in cirrhotic patients when medium chain triglycerides were substituted for long chain triglycerides in isocaloric diets. In addition, although nearly one-third of the cirrhotic patients (groups B2 and B3) showed varying degrees of malabsorption of LCT, the mean absorption rate of a medium chain fatty acid (octanoic) from a segment of upper small bowel was similar in cirrhotic and noncirrhotic patients.

Oral administration of MCT will supply the liver with considerable amounts of medium chain fatty acids. Although no unfavorable effects have been shown during prolonged administration of MCT in normal patients (24), little is known about the toxicity of MCT in cirrhotics. Two of the 12 patients developed profuse diarrhea with 
increased stool fat secretion. Eventual treatment of cirrhotic patients with MCT must await longterm controlled evaluations.

Medium chain triglycerides are known to be absorbed in the absence of lipase, not only in the form of free fatty acids and monoglycerides, but also as di- and triglycerides. Absorption of octanoic acid is apparently not dependent on pancreatic juice, since addition of pancreatic juice, as in the balloon experiments (Tables IV and V), did not alter absorption rates.

Octanoic acid has been infused into the duodenum as a clear (micellar) solution at $\mathrm{pH} 4.72(\mathrm{pK}$ octanoic acid $=4.89$ ). Since the effective $\mathrm{pK}$ of fatty acids may increase with micelle formation $(25,26)$, most of the octanoic acid may have been present in the nonionized form. Under physiologic conditions, when the $\mathrm{pH}$ of duodenal juice is approximately 6.0 to 6.5 , the ratio of ionic to nonionic octanoic acid will probably be greater than in the present study, and this may affect the rate of absorption. For example, the rate of absorption of the micellar solution of octanoic acid ( $\mathrm{pH} 4.72$ ) in one patient was $32.3 \mathrm{mg}$ per minute and only $9.8 \mathrm{mg}$ per minute when equimolar sodium octanoate $(\mathrm{pH} 6.90)$ was administered.

Since absorption rates of octanoic acid were equal in cirrhotic and noncirrhotic patients, the plasma octanoic acid levels in the various groups were of particular interest. Fasting plasma levels were negligible in all patients, but rose during perfusion of the small bowel. The finding of measurable concentrations in the plasma of noncirrhotic patients was surprising. Absorption of about $2 \mathrm{~g}$ of octanoic acid over a 60 -minute period was apparently sufficient to overcome the clearance capacity of the "normal" liver. There were, however, significant differences between cirrhotic and noncirrhotic patients and among shunted and nonshunted cirrhotic patients. With absorption of approximately equal amounts of the fatty acid (Table IV), plasma levels were significantly higher in cirrhotics, both with and without portacaval shunts, than in controls. The effect of a functional surgical portacaval shunt was clearly demonstrated by the significantly increased mean plasma levels in this group.

It was not always possible to separate patients with cirrhosis from the cirrhotic patients with a surgically constructed portacaval shunt. This is probably related to the fact that the cirrhotic patients without surgical shunts may have extensive portal-systemic anastomoses. Absorption and clearance of medium chain fatty acids may prove useful in estimating the degree of vascular shunting, whether it is pathophysiological or surgically induced. Further studies are in progress to clarify the effect of deficient liver cell function on the clearance of octanoic acid from the portal circulation.

The apparent advantages of sodium taurocholate as a nonabsorbable marker for upper small bowel studies deserve some emphasis. There was no evidence of absorption as judged by the constancy of the taurocholate to PEG concentration ratio in effluent collections. Water soluble PEG may separate from lipid phases during absorption studies (21), but micellar solutions of lipids and bile acids are much more stable. Finally, more accurate methods are available for the measurement of sodium taurocholate than for PEG.

\section{Summary}

Fat balance studies in 35 patients with alcoholic cirrhosis of the liver revealed fat malabsorption in $19(54 \%)$. Medium chain triglycerides were significantly better absorbed in a group of 10 cirrhotic patients with malabsorption of long chain triglycerides.

Absorption rates of octanoic acid from isolated segments of upper small bowel (four-lumen twoballoon technique) were similar in noncirrhotic and in cirrhotic patients, with or without portacaval shunts. One-third of these cirrhotic patients showed malabsorption of long chain triglycerides as measured by a fat balance study on a regular hospital diet containing $100 \mathrm{~g}$ fat.

Mean plasma octanoic acid levels rose from negligible fasting levels to different plateaus during perfusion of the bowel segment. Control patients showed low levels at all times, whereas a two- to threefold increase, compared to the control group, was found in the cirrhotic group, probably due to parenchymal disease and portal-systemic shunts. The effects of surgical portacaval shunts were clearly shown by a threefold increase of serum octanoic acid levels over those observed in nonoperated cirrhotic patients and by an eightfold increase over those observed in the control patients. 
Sodium taurocholate was not absorbed in the upper small bowel and may be useful as a nonabsorbable marker for fat absorption studies in the duodenum and proximal jejunum.

\section{Acknowledgments}

We extend grateful thanks to Jacqueline Glauser, Janny Brussee, Anneke Starkenburg, and Sharon Trudell for performance of the laboratory determinations; to Judith Kealy and Mary-Ann Lemelin for chemical estimations; to Anne Long and Margaret Wilcox and the nursing staff for care of the patients on the metabolic ward; to Antigone Letsou for dietary planning and management; to Gail Dardinski for preparation of the manuscript; to Rasma Klints and Dr. Hugo Muench for their assistance in the statistical analyses; and to MeadJohnson Research Center for the supply of diets and triglycerides.

\section{References}

1. Fernandes, J., J. H. van de Kamer, and H. A. Weijers. Differences in absorption of the various fatty acids studied in children with steatorrhea. J. clin. Invest. 1962, 41, 488.

2. Holt, P. R., S. A. Hashim, and T. B. Van Itallie. Treatment of malabsorption syndrome and exudative enteropathy with synthetic medium chain triglycerides. Amer. J. Gastroent. 1965, 43, 549.

3. Zurier, R. B., R. G. Campbell, S. A. Hashim, and T. B. Van Itallie. Use of medium-chain triglyceride in management of patients with massive resection of the small intestine. New Engl. J. Med. 1966, 274, 490.

4. Kiyasu, J. Y., B. Bloom, and I. L. Chaikoff. The portal transport of absorbed fatty acids. J. biol. Chem. 1952, 199, 415.

5. Playoust, M. R., and K. J. Isselbacher. Studies on the intestinal absorption and intramucosal lipolysis of a medium chain triglyceride. J. clin. Invest. 1964, 43, 878.

6. Greenberger, N. J., J. B. Rodgers, and K. J. Isselbacher. Absorption of medium and long chain triglycerides: factors influencing their hydrolysis and transport. J. clin. Invest. 1966, 45, 217.

7. Schwabe, A. D., L. R. Bennett, and L. P. Bowman. Octanoic acid absorption and oxidation in humans. J. appl. Physiol. 1964, 19, 335.

8. Valdivieso, V. D., and A. D. Schwabe. Factors influencing the absorption of a medium chain triglyceride. I. The role of bile in the intraluminal phase of absorption. Gastroenterology 1965, 48, 331.

9. Valdivieso, V. D., and A. D. Schwabe. Factors influencing the absorption of a medium chain triglyceride. II. The role of pancreatic juice in the intraluminal phase of absorption. Gastroenterology $1965,48,336$.

10. Van Goidsenhoven, G. E., W. J. Henke, J. B. Vacca, and W. A. Knight, Jr. Pancreatic function in cir- rhosis of the liver. Amer. J. dig. Dis. 1963, 8, 160.

11. Baraona, E., H. Orrego, O. Fernández, E Amenabar, E. Maldonado, F. Tag, and A. Salinas. Absorptive function of the small intestine in liver cirrhosis. Amer. J. dig. Dis. 1962, 7, 318.

12. Van de Kamer, J. H., H. ten Bokkel Huinink, and H. A. Weyers. Rapid method for the determination of fat in feces. J. biol. Chem. 1949, 177, 347.

13. Linscheer, W. G., M. S. Greenberg, E. W. Moore, and T. C. Chalmers. Studies in iron absorption. IV. Absorption in the proximal small intestine in patients with cirrhosis. Gastroenterology 1964, $46,662$.

14. Blomstrand, R., and P. Ekdahl. Fatty acid pattern of human bile under normal and pathological conditions. Proc. Soc. exp. Biol. (N. Y.) 1960, 104, 205.

15. Borgström, B., G. Lundh, and A. Hofmann. The site of absorption of conjugated bile salts in man. Gastroenterology 1963, 45, 229.

16. Hofmann, A. F. The function of bile salts in fat absorption. The solvent properties of dilute micellar solutions of conjugated bile salts. Biochem. J. 1963, 89, 57.

17. Jowett, P., and B. J. Horrocks. Improved gas chromatography of unesterified fatty acids. Nature (Lond.) 1961, 192, 966.

18. Horning, E. C., E. H. Ahrens, Jr., S. R. Lipsky, F. H. Mattson, J. F. Mead, D. A. Turner, and W. H. Goldwater. Quantitative analysis of fatty acids by gas-liquid chromatography. J. Lipid Res. 1964, 5, 20.

19. Dole, V. P., and H. Meinertz. Microdetermination of long-chain fatty acids in plasma and tissues. J. biol. Chem. 1960, 235, 2595.

20. Hyden, S. A turbidometric method for the determination of higher polyethylene glycols in biological materials. Annals of the Royal Agricultural College of Sweden 1955, 22, 139.

21. Wiggins, H. S., and A. M. Dawson. An evaluation of unabsorbable markers in the study of fat absorption. Gut 1961, 2, 373.

22. Kier, L. C. A method for the simultaneous quantitative determination of cholic acid and desoxycholic acid. I. Application to bile. J. Lab. clin. Med. 1952, 40, 755.

23. Bray, G. A. A simple efficient liquid scintillator for counting aqueous solutions in a liquid scintillation counter. Analyt. Biochem. 1960, 1, 279.

24. Hashim, S. A., A. Arteaga, and T. B. Van Itallie. Effect of a saturated medium-chain triglyceride on serum-lipids in man. Lancet 1960, 1, 1105.

25. Hofmann, A. F. A physicochemical approach to the intraluminal phase of fat absorption. Gastroenterology 1966, 50, 56.

26. Hofmann, A. F. The function of bile salts in fat absorption. Thesis, University of Lund, Sweden, 1964. 